Homology, Homotopy and Applications, vol.4(2), 2002, pp.295-314

\title{
PRESENTATION DEPTH AND THE LIPMAN-SATHAYE JACOBIAN THEOREM
}

\author{
MELVIN HOCHSTER
}

(communicated by Clas Löfwall)

\begin{abstract}
We give a version of the theorem of Lipman and Sathaye on Jacobian ideals but with substantially weaker hypotheses. Both their version and the result here are very useful in providing explicit test elements in tight closure theory. There are two separate ways in which the hypothesis in the theorem is weakened here: one is that the larger ring is not required to be a domain, although it will be reduced. Second, the regularity condition on the smaller ring is weakened to the point where one need not assume that it is Cohen-Macaulay. Instead, a condition on the ring homomorphism is imposed that may be viewed as a relative analogue of the Serre conditon $\mathrm{S}_{2}$ : a family of such conditions is introduced and studied here. The definition is made in terms of a presentation of an algebra, but is independent of the presentation.
\end{abstract}

\section{To Jan-Erik Roos on his sixty-fifth birthday}

\section{Introduction}

In this paper we shall give a version of the theorem of Lipman and Sathaye on Jacobian ideals proved in [LS] (their Theorem 2). The result presented here has substantially weaker hypotheses than in [LS]. Lipman and Sathaye used their theorem to prove the Briançon-Skoda theorem $[\mathrm{BrS}]$ (which was first proved using the analytic criterion for membership in an ideal in [Sk]; see also [LT] for background). Their result has also turned out to be very important in providing explicit test elements in tight closure theory (accounts of the latter are given in [HH1-8], [Ho2-3], $[\mathrm{Hu}]$, and $[\mathrm{Br}] ;[\mathrm{HH} 8]$, in particular, treats the use of the Lipman-Sathaye theorem in constructing test elements).

In the main theorem, Theorem (2.1) of $\S 2$ (the reader may want to consult its statement at this point), there are two separate ways in which the hypothesis in [LS] on the map $R \rightarrow S$ is weakened: first, while the $\operatorname{ring} S$ is still required to be torsion-free and generically étale over $R, S$ is not required to be a domain. Second,

The author was supported in part by a grant from the National Science Foundation.

Received March 5, 2001; published on July 12, 2002.

2000 Mathematics Subject Classification: 13B02, 13B21, $13 \mathrm{C} 15$.

Key words and phrases: Jacobian theorem, algebra presentation, depth, integral extension.

(C) 2002, Melvin Hochster. Permission to copy for private use granted. 
the regularity condition on $R$ is relaxed even to the point where we do not need to assume that $R$ is Cohen-Macaulay: a condition on the map $R \rightarrow S$ is needed, but it is significantly weaker than assuming that $R$ be Cohen-Macaulay. In this connection, we introduce in (1.2) what might be thought of as a relative analogue of the Serre condition $\mathrm{S}_{i}$ that is defined in terms of a presentation $S$ over $R$, but later shown to be independent of the presentation: cf. Theorem (1.8). It turns out that we can replace the Cohen-Macaulay condition on $R$ in the Lipman-Sathaye theorem by the condition that $S$ be relatively $\mathrm{S}_{2}$ presentable over $R$ in the sense defined in $\S 1$. A key point is that when $R \rightarrow S$ has this property, it is preserved when we adjoin an element of the normalization of $S$ : see Theorem (1.9). However, we still do need to assume that $R$ is regular when localized at a prime lying under a height one prime of the normalization of $S$.

The proof of Theorem (2.1) here is modeled on the "down-to-earth" proof of Lipman-Sathaye, and we shall make frequent references to [LS]. One difference is that we need many sources of elements in "very general position," particularly indeterminate linear combinations of generators of ideals. We are able to do this by replacing $R$ at the outset by a localization $R(t)$ of a polynomial ring in infinitely many variables over $R$.

As mentioned above, the next section is devoted to developing the required theory of "presentation $\mathrm{S}_{i}$ conditions." The main result is stated in Theorem (2.1) of $\S 2$. The proof is outlined in $\S 2$, and then the details of the various steps are supplied in $\S \S 3-5$.

\section{DEPTH PROPERTIES IN PRESENTATIONS}

Definition-Discussion 2.1. In this section fix an integer $i \geqslant 1$, let $R$ be a Noetherian domain that satisfies the Serre condition $\mathrm{S}_{i}$ (if $i=1$, the fact that $R$ is a domain guarantees this), and let $S$ be a reduced algebraic $R$-torsion-free extension of $R$ essentially of finite type over $R$. Thus, if $\mathcal{K}$ is the fraction field of $R$, then $\mathcal{K} \otimes_{R} S$ is a finite product of finite algebraic field extensions of $\mathcal{K}$. In later sections we shall assume as well that $S$ is generically étale over $R$, by which we mean that $\mathcal{K} \otimes_{R} S$ is a finite product of finite separable algebraic extensions of $\mathcal{K}$. We have that as $R$-algebras, $S \cong T / I$, where $T$ is a localized polynomial ring in finitely many variables over $R$, and we shall refer to the corresponding surjection $T \rightarrow S$ as an $L$-presentation of $S$ over $R$, where the "L-" is an indication that we are permitting $T$ to be a localization of a polynomial ring. Informally we may also say that $S \cong T / I$

is an L-presentation of $S$. An L-presentation is a presentation if $T$ is a polynomial ring in finitely many variables over $R$.

Remark 2.2. Notice that the minimal primes of $I$ all have the same height in this situation: since the elements of $R$ are nonzerodivisors in $S$, the situation is unaffected by localizing at the multiplicative system $R-\{0\}$, and thus we may assume that $R$ is a field and $S$ is a finite product of finite field extensions. Suppose that $T$ is a localization of the polynomial ring $T_{0} \subseteq T$. Then the minimal primes of $I$ must correspond to maximal ideals of $T_{0}$ (or else $S$ would transcendental over $R$ ) and so all have the same height. 
Definition 2.3. Let $R \rightarrow S$ be as in (1.0). We shall say that an L-presentation $\eta: T \rightarrow S$ with Ker $\eta=I$ is relatively $S_{i}$ if, with $h=$ ht $I$, for every prime ideal $P$ of $T$ containing $I$, depth of $T_{P} \geqslant \min \{$ ht $P, h+i\}$. (Note that this implies $\operatorname{depth}_{T} I=$ ht $I$.)

It will be convenient to have a name for the sort of ideal described above.

Definition 2.4. Let $T$ be a Noetherian ring. An ideal $I$ of $T$ all of whose minimal primes have height $h$ will be said to be of $C M_{+i}$ type if for every prime ideal $P$ containing $I, \operatorname{depth} T_{P} \geqslant \min \{$ ht $P, h+i\}$. (Again, note that this implies $\operatorname{depth}_{T} I=$ ht $I$.)

With the terminology of (1.3) and $R \rightarrow S$ as in (1.0), an L-presentation $S \cong T / I$ over $R$ is relatively $\mathrm{S}_{i}$ if and only if $I$ is of $\mathrm{CM}_{+i}$ type in $T$. Since $R$ is a domain, $T$ has no embedded primes, and (1.1) shows that the ideal $I$ associated with the presentation has the property that all of its minimal primes have the same height. We shall say that an ideal all of whose minimal primes have height $h$ has pure height $h$. Evidently, a Noetherian ring $T$ is Cohen-Macaulay if and only if every ideal of pure height is of $\mathrm{CM}_{+i}$ type for all $i$.

Although we have given Definition (1.3) without restriction on $T$, in the sequel we shall almost alway be assuming that $T$ satisfies the Serre condition $\mathrm{S}_{i}$ when we discuss ideals of $\mathrm{CM}_{+i}$ type. Likewise, in the sequel, when discussing the relatively $\mathrm{S}_{i}$ condition in (1.2), we shall almost always be assuming, as indicated in (1.0), that $R$ satisfies the Serre condition $\mathrm{S}_{i}$.

Discussion 2.5 (behavior of depth and dimension under flat base change). For use in the proof of the very important Lemma (1.5) that follows, we note two well known facts: if $(T, P) \rightarrow(W, Q)$ is a flat local homomorphism of local rings, and $B=W / P B$ is the closed fiber, then $\operatorname{dim} W=\operatorname{dim} T+\operatorname{dim} B$ ([Mat], (13.B) Theorem 19 (2), p. 79), and depth $W=\operatorname{depth} T+\operatorname{depth} B$ ([Mat], (21.C) Corollary 1, p. 154). This implies at once, for example, that if $T$ satisfies $\mathrm{S}_{i}$ and $W$ is flat over $T$ with Cohen-Macaulay fibers (or $\mathrm{S}_{i}$ fibers), then $W$ satisfies $\mathrm{S}_{i}$.

We shall make extensive use of the following alternative characterization of when an ideal $I$ is of $\mathrm{CM}_{+i}$ type.

Lemma 2.6. Let $T$ be a Noetherian ring that is $S_{i}$. Let $I \subseteq T$ be an ideal of pure height $h$. Let $f_{1}, \ldots, f_{r}$ be generators of $I$. Let $t_{\mu j}$ be indeterminates over $T$, $1 \leqslant \mu \leqslant h, 1 \leqslant j \leqslant r$, and let $g_{\mu}=\sum_{j=1}^{r} t_{\mu j} f_{j}$. Then the following are equivalent:

(1) $I$ has $C M_{+i}$ type.

(2) In some faithfully flat extension $W$ of $T, I W$ has height equal to its depth $h$, and contains a regular sequence of length $h$ generating an ideal $J$ such that $W / J$ is $S_{i}$.

(3) In the polynomial ring $W=T\left[t_{\mu j}: \mu, j\right]$, the elements $g_{1}, \ldots, g_{h}$ form a regular sequence generating an ideal $J$ such that $W / J$ is $S_{i}$.

(4) In the localization $W_{U}$ of the ring $W$ from part (3) at some (resp., every) multiplicative system $U$ consisting of polynomials whose coefficients generate the unit ideal, the elements $g_{1}, \ldots, g_{h}$ form a regular sequence generating an ideal $J$ such that $W / J$ is $S_{i}$. 
Proof. We shall prove $(3) \Rightarrow(2) \Rightarrow(1) \Rightarrow(3)$. Note that each $W_{U}$ as in (4) above is faithfully flat over $T$, and so we also have $(3) \Rightarrow((4)$ for all $U) \Rightarrow((4)$ for some $U) \Rightarrow(2)$ as well.

$(3) \Rightarrow(2)$ is obvious. To see that $(2) \Rightarrow(1)$, note that, after a faithfully flat extension $T \rightarrow W$, the ideal $I W$ has the same height as $I$ and the same depth as $I$. Thus, $I$ has height and depth $h$. Now let $P$ be a prime ideal of $R$ containing $I$ of height greater than $h$. Then there is a minimal prime $Q$ of $P W$ lying over $P$, and $T_{P} \rightarrow W_{Q}$ will be faithfully flat with a zero-dimensional fiber. The depth and dimension of $W_{Q}$ are the same as the depth and dimension of $T_{P}$ : cf. the discussion preceding (1.5). When we kill $J$ in $W_{Q}$, the depth and dimension drop by $h$, and we obtain an $S_{i}$ ring. This shows that $W_{Q}$ has the required depth, and, hence, so does $R_{P}$.

It remains to prove that $(1) \Rightarrow(3)$. The fact that the elements $g_{i}$ form a regular sequence is proved in [Ho1]. Let $Q$ be a prime containing $J=\left(g_{1}, \ldots, g_{h}\right)$ in $W=$ $T\left[t_{\mu j}: \mu, j\right]$. First suppose that $Q$ contains $I$. We must show that the depth of $W_{Q}$ is at least the smaller of ht $Q, h+i$, since this will give the correct depth once we kill $J$. Then $Q$ lies over a prime $P$ of $T$ that contains $I$. Then the depth of $R_{P}$ is at least $\min \{$ ht $P, h+i\}$, and by [Mat], (13.B) Theorem 19 (2), p. 79, we have ht $Q=$ ht $P+\operatorname{dim} B$, where $B$ is the fiber $W_{Q} / P W_{Q}$, and $\operatorname{depth} W_{Q}=\operatorname{depth} T_{P}+\operatorname{depth} B$ by [Mat], (21.C) Corollary 1, p. 154.

But the fibers of $T \rightarrow W$ are Cohen-Macaulay (in fact, regular), and so

$$
\operatorname{depth} W_{Q} \geqslant \min \{\text { ht } P, h+i\}+\operatorname{depth} B=\min \{\text { ht } P, h+i\}+
$$

$\operatorname{dim} B \geqslant \min \{$ ht $P+\operatorname{dim} B, h+i+\operatorname{dim} B\}=\min \{$ ht $Q, h+i+\operatorname{dim} B\} \geqslant$ $\min \{$ ht $Q, h+i\}$, as required.

It remains only to consider the case where $Q$ fails to contains $I$. Then one of the generators $f_{j}$ of $I$ is a unit in $T_{P}$, where $P$ is the contraction of $Q$ to $T$. Again, we pass to $T_{P}$, and renumber so that $f_{r}$ is a unit. Then the equations $g_{\mu}$ that we are killing simply solve for the indeterminates $t_{\mu r}$ in terms of the others, and the quotient by $J$ may be identified with $T_{P}\left[t_{\mu j}: 1 \leqslant \mu \leqslant h, 1 \leqslant j<r\right]$, a polynomial ring over $T_{P}$. Since this ring is $\mathrm{S}_{i}$ so is the polynomial ring, and the result follows.

We shall later need the following fact whose proof is really a variant of the argument that $(1) \Rightarrow(3)$ in Lemma (1.5) above.

Lemma 2.7. Let $I$ be an ideal of pure height $h$ in a Noetherian ring $T$ such that $T$ is $S_{i}$. Let $u_{1}, \ldots, u_{h}$ be a regular sequence in $I$ such that $T /\left(u_{1}, \ldots, u_{h}\right) T$ is $S_{i}$. Let $f_{1}, \ldots, f_{r}$ be generators of $I$. Let $k$ be an integer, $1 \leqslant k \leqslant h$. Let $t_{\mu j}$ be indeterminates over $T, 1 \leqslant \mu \leqslant k, 1 \leqslant j \leqslant h$ and let $t_{\mu j}^{\prime}$ also be indeterminates over $T, k<\mu \leqslant h, 1 \leqslant j \leqslant r$. Let $W$ be the ring obtained by adjoining all these independent indeterminates to $T$. Then the $k$ elements $g_{\mu}=\sum_{j=1}^{h} u_{j} t_{\mu j}, 1 \leqslant \mu \leqslant k$, together with the $h-k$ elements $g_{\mu}=\sum_{j=1}^{r} f_{j} t_{\mu j}^{\prime}, k<\mu \leqslant h$, give a regular sequence in $I W$ such that the quotient of $W$ by the ideal they generate is $S_{i}$. 
Proof. Consider a prime $Q$ of $W$ that contains the $g$ 's. The specified depth condition on $W_{Q} /\left(g_{1}, \ldots, g_{h}\right)$ is equivalent to the condition that the depth of $W_{Q}$ be at least the lesser of ht $Q, h+i$.

If $Q$ contains all the elements $u_{1}, \ldots, u_{h}$ this follows because, after we kill this regular sequence, the quotient ring $W_{Q} /\left(u_{1}, \ldots, u_{h}\right)$ is $S_{i}$, since it is flat over $T /\left(u_{1}, \ldots, u_{h}\right)$ with regular fibers. If $Q$ fails to contain all the $u_{h}$, we replace $R$ by its localization at the contraction $P$ of $Q$ to $T$. We may assume that in each of the first $k$ of the $g_{\mu}$, one of the $u_{i}$ is invertible, permitting us to use $g_{\mu}=0$ to solve for one of the $t_{\mu j}$ in terms of the others. But then $P \nsupseteq I$ as well, and so in each of the last $n-k$ of the $g_{\mu}$ at least one of the $f_{j}$ is invertible, permitting us to solve for one of the $t_{\mu j}$ in terms of the others. This means that the quotient by $\left(g_{1}, \ldots, g_{h}\right)$ may be identified with a polynomial ring over $T_{P}$, and the result follows from the fact that $T$ is $\mathrm{S}_{i}$.

Note that if $R$ is Cohen-Macaulay then so is $T$, and it is then automatic that every L-presentation is relatively $\mathrm{S}_{i}$ for all $i$.

Our next results are aimed at showing that when $S$ has a relatively $S_{i}$ presentation over $R$, then every presentation of $S$ over $R$ is relatively $S_{i}$.

Lemma 2.8. Let $R \rightarrow S$ be a homomorphism as in (1.0). Let $S \cong T / I$ be an $L$-presentation of $S$ as an $R$-algebra that is relatively $S_{i}$, and let $T_{0}$ be a polynomial ring in finitely many variables over $R$ contained in $T$ such that $T$ is a localization of $T_{0}$.

(a) If $U$ is a multiplicative system in $T$ with image $V$ in $S$ then $T_{U} / I_{U}$ is a relatively $S_{i}$ L-presentation of $S_{V}$ over $R$.

(b) (Descent of localizations.) Assume that the $S_{i}$ locus is open in rings essentially of finite type over $R$ (which holds if $R$ is either a homomorphic image of a CohenMacaulay ring or if $R$ is excellent). If $T=\left(T_{0}\right)_{U}$ for a multiplicative system $U$ in $T_{0}$, there is an element $u \in U$ such that if $T_{1}=T_{0}[1 / u]$, and $I_{1}=I \cap T_{1}$, then $S_{1}=T_{1} / I_{1}$ is a relatively $S_{i} L$-presentation.

Proof. Part (a) is clear, since the set of localizations at primes containing $I$ can only become smaller as we localize $T$. It is worth noting that the condition that $I$ have pure height is needed here: otherwise, the height of $I$ might increase after localization.

To prove part (b), note that localizing at finitely many elements is equivalent to localizing at their product. We may make several choices of $u, T_{1}$, each time localizing at one more element to get additional conditions to hold. Choose generators $f_{1}, \ldots, f_{r}$ for $I$ and initially choose $u$ so that these will be in $T_{1}$. Thus, we may assume that $I=I_{1} T$. Let $h=\mathrm{ht} I$. Introduce $h r$ new indeterminates $t_{\mu j}$ and define $g_{1}, \ldots, g_{h}$ as in part (3) of Lemma (1.5). Then we know that $U^{-1} T_{1}\left[t_{\mu j}: \mu, j\right] /\left(g_{1}, \ldots, g_{h}\right)$ is $S_{i}$, by Lemma (1.5). This means that $U$ meets the defining radical ideal of the non-S $S_{i}$ locus in the ring $T_{1}\left[t_{\mu j}: \mu, j\right] /\left(g_{1}, \ldots, g_{h}\right)$ (our hypothesis guarantees that this locus is closed). Thus, we may choose $u_{1} \in U$ meeting that radical ideal. Replacing $T_{1}$ by its localization at $u_{1}$, we see that we can localize at just one element and get $T_{1}\left[t_{\mu j}: \mu, j\right] /\left(g_{1}, \ldots, g_{h}\right)$ to be $\mathrm{S}_{i}$ (this is 
a new choice of $T_{1}$ ), and so by part (3) of Lemma (1.5), $T_{1} \rightarrow T_{1} / I_{1}$ is relatively $\mathrm{S}_{i}$ for this new choice of $T_{1}$.

Theorem 2.9 ((independence of presentation)). Let $R \rightarrow S$ be a homomorphism as in (1.0) and suppose that $S$ has an L-presentation over $R$ that is relatively $S_{i}$. Then every L-presentation of $S$ over $R$ is relatively $S_{i}$.

Proof. Step 1. We first consider the case where $S$ is finitely generated over $R$ and we are considering only presentations of $S$ over $R$, i.e., $T$ is required to be a polynomial ring. Given two presentations $T \rightarrow S, T^{\prime} \rightarrow S$, we can compare both with $T \otimes_{R}$ $T^{\prime} \rightarrow S$. By induction on the number of variables in $T^{\prime}$, we reduce to considering a presentation $T \rightarrow S$ and another $T[X] \rightarrow S$, where $X$ is a new indeterminate. Let $s$ be the image of $X$ and let $z \in T$ map to $s$. We may replace $X$ by $X-z$, and so assume that $X$ maps to 0 in $S$. Thus, if $I=\operatorname{Ker}(T \rightarrow S)$, the kernel of the second presentation will be $I T[X]+X T[X]$. The primes of $T[X]$ containing the kernel are in bijective correspondence with the primes of $T$ containing $I$, and the depth condition is obviously satisfied for $T_{P}$ if and only if it is satisfied for $T[X]_{Q}$, where $Q=P T[X]+X T[X]$ is the corresponding prime, since $T[X]_{Q} /(X) \cong T_{P}$, and $X$ is a nonzerodivisor.

Step 2. We next consider the case where $S$ is finitely generated over $R$, but can be written $S=S_{0}[1 / s]$ where $s \in S_{0}$. Let $T_{0} \rightarrow S_{0}$ be a presentation of $S_{0}$ with kernel $I_{0}$ and and $v \in T_{0}$ an element that maps to $s$. Then we have a surjection $T_{0}[1 / v] \rightarrow S$, which is an L-presentation, and we also have a presentation $T_{0}[X] \rightarrow S$ extending $T_{0} \rightarrow S_{0}$, where $X$ maps to $1 / s$. The kernel of the latter is $I_{1}=I_{0} T_{0}[X]+(X v-1)$. Again, there is a bijection between the primes of $T_{0}[1 / v]$ containing $I_{0}$ (or primes of $T_{0}$ containing $I_{0}$ and not $v$ ) and the primes of $T_{0}[X]$ containing $I_{1}$, and the depths in the localizations at the latter primes are all one greater: we can see this, because we can start by killing $X v-1$, which gives the correspondence. Thus an L-presentation of this type is relatively $S_{i}$ presentable if and only if the corresponding presentation is relatively $\mathrm{S}_{i}$ presentable.

Step 3. We now consider the general case. Let $T_{0}$ be a polynomial ring in finitely many variables over $R$ and $U$ a multiplicative system in $T_{0}$ such that $U^{-1} T_{0} \rightarrow S$ is a relatively $\mathrm{S}_{i}$ L-presentation of $S$ over $R$. By Lemma (1.7) (b) we can choose a localization $T_{1}$ of $T_{0}$ at one element and such that the restriction of $T \rightarrow S$ gives a surjection $T_{1} \rightarrow S_{1}$ that is a relatively $\mathrm{S}_{i}$ L-presentation, and $S$ is a localization of $S_{1}$. By part (a), every localization $S_{2}$ of $S_{1}$ at one element has an L-presentation $T_{2} \rightarrow S_{2}$ that is relatively $S_{i}$ and such that $T_{2}$ is a localization of a polynomial ring at one element. It follows that $S$ is a localization of a finitely generated $R$-subalgebra $S_{1}$ with the following property: every presentation of $S_{1}$, and every presentation of any localization $S_{1}[1 / u]$, is relatively $\mathrm{S}_{i}$.

Now consider some other L-presentation $\left(U^{\prime}\right)^{-1} T^{\prime} \rightarrow S$. Then we can choose a localization $T_{1}^{\prime}$ of $T^{\prime}$ at one element of $U^{\prime}$ whose image $S_{1}^{\prime}$ in $S$ contains $S_{1}$, and we can choose $w \in S_{1} \subseteq S_{1}^{\prime}$ such that $S_{1}^{\prime}[1 / w]=S_{1}[1 / w]$, where $w$ is invertible in $S$. Thus, $S_{1}^{\prime}[1 / w]$ has a presentation that is relatively $\mathrm{S}_{i}$.

Suppose that $w=\tau / y$ where $\tau \in T^{\prime}$ and $y \in U^{\prime}$. Since $w$ is invertible in $S$, we can choose $a \in T^{\prime}$ and $z \in U^{\prime}$ such that $a \tau / y z=1$ in $S$, i.e., and then there exists $v \in U^{\prime}$ such that $v a \tau=v y z$. Then $S_{1}^{\prime}$ localized at the image $\zeta$ of $v y z$ contains $1 / w$, since 
that can be represented as $a / z$, and so $S_{2}=S_{1}^{\prime}[1 / \zeta]$ has the property that every presentation is relatively $\mathrm{S}_{i}$, since it is a localization of $S_{1}[1 / w]$ at one element, and hence a localization of $S_{1}$ at one element. But $T_{1}^{\prime}[1 / v y z]$ is a localization of $T^{\prime}$ at an element of $U^{\prime}$ mapping onto $S_{2}$. From Step 2 we know that this L-presentation is also relatively $\mathrm{S}_{i}$ (since the corresponding presentation will be), and then by (1.7) (a), $\left(U^{\prime}\right)^{-1} T^{\prime} \rightarrow S$ is relatively $\mathrm{S}_{i}$.

The following fact will be critical in the proof of the Lipman-Sathaye Jacobian theorem.

Theorem 2.10. Let $R \rightarrow S$ be as in (1.0). Suppose that $S$ is relatively $S_{i}$ presentable over $R$. Let $s$ be an element of the total quotient ring of $S$ that is integral over $S$. Then $S[s]$ is relatively $S_{i}$ presentable over $R$. Hence, any subring of the integral closure of $S$ module-finite over $R$ is relatively $S_{i}$ presentable over $R$. Moreover, if $T \rightarrow R$ is an L-presentation with kernel $I$ of height $h$ such that $g_{1}, \ldots, g_{h}$ is a regular sequence in $I$ with the property that $T /\left(g_{1}, \ldots, g_{h}\right)$ is $S_{i}$, and we extend $T \rightarrow S$ to $T[X] \rightarrow S[s]$ by sending $X$ to $s$, then there is a polynomial $F$ monic in $X$ in the kernel, and $T[X] /\left(g_{1}, \ldots, g_{h}, F\right)$ is $S_{i}$.

Proof. We first establish the last statement. Let $B=T /\left(g_{1}, \ldots, g_{h}\right)$ and

$$
C=T[X] /\left(g_{1}, \ldots, g_{h}, F\right) \cong B[X] /(f)
$$

where $f$ is the image of $F$ in $B[X]$ and is still monic in $X$. Clearly, $B[X] /(f)$ is flat (and even free) over $B$ with zero-dimensional (hence, Cohen-Macaulay) fibers, and so is $\mathrm{S}_{i}$.

The first part now follows. After tensoring with $R, S, T R\left[t_{\mu j}: \mu, j\right]=R[t]$ we get such a regular sequence in $T[t]$ such that the quotient is $\mathrm{S}_{i}$, by Lemma (1.5) (3). When we adjoin $X$ the result of the paragraph above together with Lemma (1.5) (2) shows that we have a relatively $\mathrm{S}_{i}$ presentation of $S[s]$.

The following fact is used in Discussion (1.11) to show that there are many case where $R \rightarrow S$ as in (1.0) is such that $S$ is relatively $\mathrm{S}_{2}$ presentable over $R$, but $R$ is not Cohen-Macaulay.

Proposition 2.11. Let $R \rightarrow S$ be as in (1.0) (in particular, $R$ satisfies $S_{i}$ ) and let $R_{1}$ be a domain flat over $R$ with $S_{i}$ fibers. Suppose that $S$ is relatively $S_{i}$ presentable over $R$. Then $R_{1} \rightarrow R_{1} \otimes_{R} S$ satisfies (1.0) and is relatively $S_{i}$ presentable over $R_{1}$.

Proof. Let $T \rightarrow S$ be an L-presentation (with kernel $I$ of pure height $h$ )that is relatively $\mathrm{S}_{i}$. After adjoining indeterminates $t_{\mathfrak{m} j}$ as in (1.5) we obtain a regular sequence $g_{1}, \ldots, g_{h}$ in the expansion of $I$ such that $T[t] /\left(g_{1}, \ldots, g_{h}\right)$ is $\mathrm{S}_{i}$. The main point is that $R_{1} \otimes_{R} T[t] /\left(g_{1}, \ldots, g_{h}\right)$ is $\mathrm{S}_{i}$. Cf. (1.4).

Discussion 2.12. We conclude this section with an example which shows that the property that $S$ be relatively $\mathrm{S}_{2}$ presentable over $R$ is weaker than the assumption that $R$ be Cohen-Macaulay.

One way to see this is to let $R_{0}$ be Cohen-Macaulay normal of finite type over an algebraically closed field $K$, and let $B$ denote a finite type $K$-algebra that is 
normal but not Cohen-Macaulay. Take any extension of $S_{0}$ of $R_{0}$ as in (1.0). This will be relatively $S_{2}$-presentable simply because $R_{0}$ is Cohen-Macaulay. It is easy to see that $R=B \otimes_{K} R_{0} \rightarrow B \otimes_{K} S_{0}=S$ is still relatively $\mathrm{S}_{2}$ presentable (apply (1.10) with $R=R_{0}$ and $R_{1}=B \otimes_{K} R_{0}$ ), but $R$ is not Cohen-Macaulay.

\section{STATEMENT OF THE THEOREM AND A SKETCH OF THE PROOF}

Throughout the rest of this paper $R$ denotes a Noetherian domain with fraction field $\mathcal{K}$, and $S$ denotes an algebra essentially of finite type over $R$ (i.e., a localization at some multiplicative system of a finitely generated $R$-algebra) such that $S$ is torsion-free and generically étale over $R$ (by which we mean that $\mathcal{L}=\mathcal{K} \otimes_{R} S$ is a finite product of finite separable algebraic field extensions of $\mathcal{K}$ ). We shall denote by $S^{\prime}$ the integral closure of $S$ in $\mathcal{L}$. We shall see that $S^{\prime}$ is module-finite over $S$ if $R$ is regular or excellent (cf. (2.7)).

If $A$ and $B$ are subsets of $\mathcal{L}$ we denote by $A:{ }_{\mathcal{L}} B$ the set $\{u \in \mathcal{L}: u B \subseteq A\}$. If $T$ is a subring of $\mathcal{L}$ and $A$ is a $T$-module, then so is $A:{ }_{\mathcal{L}} B$.

We shall write $J_{S / R}$ for the Jacobian ideal of $S$ over $R$. If $S$ is a finitely generated $R$-algebra, so that we may think of $S$ as $R\left[X_{1}, \ldots, X_{n}\right] /\left(f_{1}, \ldots, f_{m}\right)$, then $J_{S / R}$ is the ideal of $S$ generated by the images of the size $n$ minors of the Jacobian matrix $\left(\partial f_{j} / \partial x_{i}\right)$ under the surjection $R[X] \rightarrow S$. This turns out to be independent of the presentation. Moreover, if $s \in S$, then $J_{S_{s} / R}=J_{S / R} S_{s}$. From this one sees that if $S$ is essentially of finite type over $R$ and one defines $J_{S / R}$ by choosing a finitely generated subalgebra $S_{0}$ of $S$ such that $S=W^{-1} S_{0}$ for some multiplicative system $W$ of $S_{0}$, and takes $J_{S / R}$ to be $J_{S_{0} / R} S$, then $J_{S / R}$ is independent of the choices made. The result we aim to prove is:

Theorem 3.1 (Generalized Lipman-Sathaye Jacobian theorem). Let $R$ be a Noetherian domain with fraction field $\mathcal{K}$. Assume also that the $S_{2}$ locus is open in algebras essentially of finite type over $R .^{1}$ Let $S$ be an extension algebra essentially of finite type over $R$ such that $S$ is torsion-free and generically étale over $R$. Suppose that for every maximal ideal $\mathcal{M}$ of $S$ with contraction $\mathcal{P}$ to $R, R_{\mathcal{P}}$ is normal, and $S_{\mathcal{M}}$ has a relatively $S_{2}$ presentation over $R_{\mathcal{P}} .{ }^{2}$ Let $\mathcal{L}=\mathcal{K} \otimes_{R} S$ and let $S^{\prime}$ be the integral closure of $S$ in L. Assume that $S^{\prime}$ is module-finite over $S .{ }^{3}$ Suppose that for every height one prime ideal $Q$ of $S^{\prime}, R_{Q \cap R}$ is regular. Then $S^{\prime}:{ }_{\mathcal{L}} J_{S^{\prime} / R} \subseteq S:{ }_{\mathcal{L}} J_{S / R}$.

\section{Overview of the Proof.}

(2.2) Step 1: The local case suffices Note that it is enough to prove the result when $S$ is replaced by its various localizations at maximal ideals. Thus, we may assume that $S$ is local, although we shall only make this assumption at certain

\footnotetext{
${ }^{1}$ This holds automatically if $R$ is excellent or if $R$ is a homomorphic image of a Cohen-Macaulay ring.

${ }^{2}$ This is automatic if $R$ is normal and Cohen-Macaulay, or if $R$ is normal and $S$ has a relatively $\mathrm{S}_{2}$ presentation over $R$.

${ }^{3}$ This is automatic if $R$ is regular or excellent: cf. (2.7).
} 
points in the proof. When $S$ is local we may also replace $R$ by its localization at the contraction of the maximal ideal of $S$, and so there is likewise no loss of generality in assuming that $R$ is local and that $R \rightarrow S$ is local homomorphism (i.e., the maximal ideal of $R$ maps into that of $S$ ). Because of this reduction to the local case, throughout the rest of the argument we assume that $R$ is a normal local domain and, in particular, that $R$ satisfies $\mathrm{S}_{2}$.

(2.3) Step 2: Adjoining indeterminates If $\mathcal{T}=\left\{t_{\lambda}: \lambda \in \Lambda\right\}$ is a family of indeterminates over a Noetherian ring $R$ we denote by $R(\mathcal{T})$ or sometimes even $R(t)$ the localization of the polynomial ring $R\left[t_{\lambda}: \lambda \in \Lambda\right]$ at the multiplicative system consisting of all polynomials whose coefficients generate the unit ideal. This ring is Noetherian even when the family of indeterminates is infinite (cf. [HH7], Remark 2.18, especially the final, parenthetical paragraph) and faithfully flat over $R$ with geometrically regular fibers, since it is a direct limit of smooth $R$-algebras and flat maps and happens to be Noetherian. To prove Theorem (2.2) it suffices to consider $R(t) \rightarrow R(t) \otimes_{R} S$ instead of $R \rightarrow S$. Note that the normalization of $R(t) \otimes_{R} S$ is $R(t) \otimes_{R} S^{\prime}$ : it is normal because it is a localization of a polynomial ring over $S^{\prime}$. Note that the map $R(t) \rightarrow R(t) \otimes_{R} S$ is the direct limit of maps $R\left(\mathcal{T}_{0}\right) \rightarrow R\left(\mathcal{T}_{0}\right) \otimes_{R} S$ as $\mathcal{T}_{0}$ runs through finite subsets of $\mathcal{T}$.

Note that if $R$ has the property that algebras essentially of finite type over $R$ have open $\mathrm{S}_{i}$ locus, then so does $R(t)$. It suffices to see this for algebras of finite type over $R(t)$ and each of these arise from a finite type algebra $S_{0}$ over $R\left(\mathcal{T}_{0}\right)$ (where $\mathcal{T}_{0}$ is finite) by a base change $R\left(\mathcal{T}_{0}\right) \rightarrow R(t)$ that is faithfully flat with regular fibers. But then $Q$ in $R(t) \otimes_{R\left(\mathcal{T}_{0}\right)} S_{0}$ with contraction $P$ to $S_{0}$ is such that the localization at $Q$ is $\mathrm{S}_{i}$ if and only if $\left(S_{0}\right)_{P}$ has property $\mathrm{S}_{i}$, i.e., the locus that we want in Spec $\left(R(t) \otimes_{R\left(\mathcal{T}_{0}\right)} S_{0}\right)$ is the inverse image of an open set in Spec $S_{0}$ (since $S_{0}$ is essentially of finite type over $R$ ).

Note also that if $R \rightarrow S$ has the property that every height one prime of $S^{\prime}$ lies over a prime $P$ in $R$ such that $R_{P}$ is regular, the same is true for $R(t) \rightarrow R(t) \otimes_{R} S$. Since the latter ring is flat over $S$, a height one prime there lies over either a height one or height 0 prime of $S$ : in either case, that in turn lies over a prime $P_{0}$ in $R$ such that $R_{P_{0}}$ is regular (if the contraction to $S$ is height 0 , the prime lies over 0 in $R$ ). Thus, $R(t)_{P}$ will be a Noetherian localization of a polynomial ring over $R_{P_{0}}$, and, hence, regular.

Thus, we may replace $R \rightarrow S$ by $R(t) \rightarrow S(t)$ in proving the theorem, where the set of indeterminates is infinite. This will prove useful in constructing regular sequences in sufficiently general position.

(2.4) Step 3: Presenting $\boldsymbol{S}$ over $\boldsymbol{R}$ Let $T$ denote a localization of $R\left[X_{1}, \ldots, X_{n}\right]$ that maps onto $S$, and let $I$ denote the kernel. Let $U$ denote the complement in $T$ of the set of minimal primes $P_{1}, \ldots, P_{r}$ of of $I$ in $T$. Since $S$ is reduced, $I=\bigcap_{i=1}^{r} P_{i}$. Since $S$ is a torsion-free $R$-module, the minimal primes of $I$ do not meet $R$, and correspond to the minimal primes of $I(\mathcal{K} \otimes T)$. Since killing any of these minimal primes produces an algebraic extension of $\mathcal{K}$, they must correspond to maximal ideals of $\mathcal{K}\left[X_{1}, \ldots, X_{n}\right]$, and it follows that the $P_{i}$ all have the same height, which must be the same as the number of variables, $n$. Thus, $U^{-1} T$ is a semilocal regular ring in which each of the maximal ideals $\mathcal{M}_{i}=P_{i} U^{-1} T$ is generated by $n$ elements. 
(2.5) Step 4: Special sequences and the modules $W_{S / R}$ Call a sequence $g_{1}, \ldots, g_{n}$ of $n$ elements of $I$ special if it generates each of the $\mathcal{M}_{i}$, is a regular sequence in $T$, and all of the associated primes of the ideal it generates are of height $n$. We shall show that special sequences exist (this is really only using that $S$ has a relatively $\mathrm{S}_{1}$ presentation over $R$ ), and that there are sufficiently many of them that the images of the elements $\operatorname{det}\left(\partial g_{j} / \partial X_{i}\right)$ in $S$ with $g_{1}, \ldots, g_{n}$ special generate the Jacobian ideal. Moreover, when $g_{1}, \ldots, g_{n}$ is special the image of $\operatorname{det}\left(\partial g_{j} / \partial X_{i}\right)$ in $S$ is not a zerodivisor in $S$, and so has an inverse in $\mathcal{L}$. Given $\theta: T \rightarrow S$ and a special sequence $g_{1}, \ldots, g_{n}$ we define a map

$$
\Phi: \frac{\left(g_{1}, \ldots, g_{n}\right) T:{ }_{T} I}{\left(g_{1}, \ldots, g_{n}\right) T} \rightarrow \mathcal{L}
$$

by sending the class of $u$ to $\bar{u} / \gamma$, where $\bar{u}$ is the image of $u$ in $S$ and $\gamma$ is the image of $\operatorname{det}\left(\partial g_{j} / \partial X_{i}\right)$ in $S$. We shall show that $\Phi$ is injective. A priori, its image depends on the choice of $T \rightarrow S$ and on the choice of the special sequence $g_{1}, \ldots, g_{n}$, but the image turns out to be independent of these choices, and so we have constructed a finitely generated canonically determined $S$-module $W_{S / R} \subseteq \mathcal{L}$.

(2.6) Step 5: The main idea of the argument It will turn out that, quite generally, $W_{S / R} \subseteq S:{ }_{\mathcal{L}} J_{S / R}$. The result then follows from two further observations. The first is that when $S$ is normal, this is an equality. (The idea is to show that $W_{S / R}$ is then $\mathrm{S}_{2}$, which forces $\Phi$ with its image restricted to $S:{ }_{\mathcal{L}} J_{S / R}$ to be an isomorphism - this can be verified after localizing at height one primes of $S$. This is the only place in the proof where we use the hypothesis that $S$ has a relatively $\mathrm{S}_{2}$ presentation over $R$.) The second is that when one enlarges $S$ to $S_{1}=S\left[s_{1}\right]$ by adjoining one integral fraction $s_{1} \in \mathcal{L}$ (so that $S \subseteq S_{1} \subseteq S^{\prime}$ ), then $W_{S_{1} / R} \subseteq W_{S / R}$. Repeated application of this fact yields that $W_{S^{\prime} / R} \subseteq W_{S / R}$ and then we have

$$
S^{\prime}:{ }_{\mathcal{L}} J_{S^{\prime} / R}=W_{S^{\prime} / R} \subseteq W_{S / R} \subseteq S:{ }_{\mathcal{L}} J_{S / R},
$$

and we are done. In the sequel we shall systematically fill in the details of this outline.

We conclude this section with a result, essentially in [LS] but not stated in this generality, which shows that the normalization $S^{\prime}$ of $S$ is module-finite over $S$ under very mild hypotheses on $R$.

Fact 3.2 (finiteness of the normalization). Let $S$ be torsion-free, generically étale, and essentially of finite type over a normal Noetherian domain $R$. Suppose that the completion of every local ring of $R$ is reduced (which holds if $R$ is either regular or excellent). Then the normalization $S^{\prime}$ of $S$ over $R$ is module-finite over $S{ }^{4}$

\footnotetext{
${ }^{4}$ Sketch of proof. Let $S_{0} \in S$ be finite type over $R$ with $S=\left(S_{0}\right)_{W}$. Since localization commutes with normalization, we may replace $S$ by $S_{0}$ and assume $S$ is finite type over $R$. The integral closure of $S$ is the product of the integral closures of the domains obtained by killing a minimal prime of $S$. Hence, we may assume $S$ is a domain. Each of finitely many generators for $S$ over $R$ satisfies an algebraic equation over $R$ with leading coefficient $r_{\nu}$, say, and so with $r=\prod_{\nu} r_{\nu} \in R-\{0\}$, $S[1 / r]$ is integral over $R[1 / r]$. The integral closure of the normal domain $R[1 / r]$ in the fraction field $\mathcal{L}$ of $S[1 / r]$ is the same as the normalization of $S[1 / r]$, and is module-finite over $S[1 / r]$ by Prop. (31.B) of Chapter 12 of [Mat]. We may enlarge $S$ by adjoining finitely many elements of its normalization and so obtain a domain $S$ such that $S[1 / r]$ is normal for some $r \neq 0$. By Lemma 4
} 


\section{BEGINNING THE PROOF: EXISTENCE OF SPECIAL SEQUENCES}

Throughout the rest of this paper $R$ denotes a normal Noetherian domain with fraction field $\mathcal{K}$, and $S$ denotes an algebra essentially of finite type over $R$ (i.e., a localization at some multiplicative system of a finitely generated $R$-algebra) such that $S$ is torsion-free and generically étale over $R$ (by which we mean that $\mathcal{L}=$ $\mathcal{K} \otimes_{R} S$ is a finite product of finite separable algebraic field extensions of $\mathcal{K}$ ). We shall denote by $S^{\prime}$ the integral closure of $S$ in $\mathcal{L}$, and we shall assume that it is a finite module over $S$. We also assume that $S$ is relatively $\mathrm{S}_{1}$ presentable over $R$. (We shall not need that it is relatively $\mathrm{S}_{2}$ presentable until $\S 4$.) Let $T \rightarrow S$ be an Lpresentation with kernel $I$ of pure height $n$. Recall that a regular sequence $g_{1}, \ldots, g_{n}$ of length $n$ in $I$ is special if $g_{1}, \ldots, g_{n}$ generate $I T_{P}$ for every minimal prime $P$ of $I$, and all minimal primes of $\left(g_{1}, \ldots, g_{n}\right) T$ have height $n$. We construct many special sequences after replacing $R, S, T$ by their tensor products over $R$ with $R(\mathcal{T})$, where $\mathcal{T}$ is consists of infinitely many new indeterminates as in (2.3). We denote the results of tensoring $R, S, T$ with $R(\mathcal{T})=R_{1}$ by $R_{1}, S_{1}$, and $T_{1}$, respectively. We write $I_{1}$ for the expansion of $I$ to $T_{1}$, so that $S_{1} \cong T_{1} / I_{1}$. In later sections we shall change notation and write $R, S, T$ for the rings denoted $R_{1}, S_{1}, T_{1}$ here.

Lemma 4.1. With hypothesis as in the paragraph above, there are sufficiently many special sequences $g_{1}, \ldots, g_{n}$ in $I_{1}$ that the Jacobian ideal $J_{S_{1} / R_{1}}$ is generated by the images of elements $\operatorname{det}\left(\partial g_{j} / \partial X_{k}\right)$ for $g_{1}, \ldots, g_{n}$ special (where the $X_{k}$ are the indeterminates generating the polynomial ring of which $T$ is the localization). Moreover, if $T_{1}$ is local, given any two special sequences there is a finite sequence (which we shall refer to as a chain) of special sequences with one of them as first term and the other as last term with the following property: given any two consecutive terms, one is obtained from the other by letting an invertible matrix over $R_{1}$ act, or else the two consecutive sequences differ in only one term.

Proof. Pick generators $f_{1}, \ldots, f_{r}$ for $I$. Let $\left[t_{\mu j}\right]$ be an $r \times r$ matrix of indeterminates from the infinite set $\mathcal{T}$. The elements $g_{\mu}=\sum_{j=1}^{r} f_{j} t_{\mu j}$ give a new set of generators for $I_{1}$, because the determinant of $\left[t_{\mu j}\right]$ is invertible in $R_{1}$. By Lemma (1.5) applied with $i=1$, any $n$ of these generators form a regular sequence $W=T\left[t_{\mu j}: \mu j\right]$ such that the ideal they generate has no embedded primes (the last follows from the fact that the quotient is $\mathrm{S}_{1}$ ). Because the map $W \rightarrow T_{1}$ is flat with Cohen-Macaulay fibers, the sequence retains these properties. Next, we want check that any $n$ of these generate after localizing at a minimal prime $P$ of $I_{1}$. Since they generate $I_{1}$,

of Ch. 12 of [Mat], $S$ has finite normalization if $S_{Q}$ does for every maximal ideal $Q$ of $S$. Choose $s \in S$ with $\mathcal{L}=\mathcal{K}[s]$. Let $R_{1}=R[s]$ and $P=Q \cap R_{1}$. Then $S_{Q}$ is a localization of $\left(R_{1}\right)_{P}[S]$, and $S$ is generated over $\left(R_{1}\right)_{P}$ by elements of its fraction field. By a result of [Rees], if the completion of a local domain $B$ is reduced, then the normalization of any algebra $C$ finitely generated over $B$ by fractions is module-finite over $C$. Thus, it suffices to show that the completion of $\left(R_{1}\right)_{P}$ is reduced. We may replace $R$ by its localization at the contraction of $P$, and so we may assume that $(R, m)$ is local with reduced completion. The completion of $\left(R_{1}\right)_{P}$ is one of the local rings of the completion of $R_{1}$ with respect to $m$. Thus, it suffices if this completion of $R_{1}$ is reduced. But this is $R_{1} \otimes_{R} \widehat{R} \subseteq \mathcal{L} \otimes_{R} \widehat{R} \cong \mathcal{L} \otimes_{\mathcal{K}}\left(\mathcal{K} \otimes_{R} \widehat{R}\right)$, and the result follows because $\mathcal{K} \otimes_{R} \widehat{R}$ is reduced and $\mathcal{L} / \mathcal{K}$ is separable. 
it is certainly true that we can choose $n$ of them to generate $I_{1}\left(T_{1}\right)_{P}$. Given the symmetric roles played by the indeterminates, it must be true that any $n$ generate. Since we may use all $n$ elements subsets of this set of generators of $I_{1}$, we obtain enough elements to generate the Jacobian ideal.

Now suppose that we have two special sequences in $I_{1}$. They only involve finitely many of the indeterminates in $\mathcal{T}$. After replacing $R$ by $R\left(\mathcal{T}_{0}\right)$ for a suitable finite subset $\mathcal{T}_{0}$ of $\mathcal{T}$, and making corresponding changes in $S, T$, we may assume without loss of generality that the two special sequences are in $T$.

We choose $n^{2}+n r$ new indeterminates from $\mathcal{T}$ to construct two $n \times n$ matrices $\alpha, \beta$ of indeterminates from $\mathcal{T}$ as well as an $n \times r$ matrix $\gamma$. We replace one special sequence by letting $\alpha$ act on it: call it $a_{1}, \ldots, a_{n}$. We replace the other by letting $\beta$ act on it: call the result $b_{1}, \ldots, b_{n}$. Also let $\gamma$ act on the $f_{1}, \ldots, f_{r}$ to form $n$ linear combinations, say $g_{1}, \ldots, g_{n}$, of the $f_{1}, \ldots, f_{r}$ with indeterminate coefficients. If we replace the terms in $a_{1}, \ldots, a_{n}$ one at a time by the $f$ 's, we make a chain from $a_{1}, \ldots, a_{n}$ to $f_{1}, \ldots, f_{n}$. We can then reverse the process to make a chain from $f_{1}, \ldots, f_{n}$ to $b_{1}, \ldots, b_{n}$, changing only one term at a time. The fact that each of the sequences formed is a regular sequence generating an ideal with no embedded primes is a consequence of Lemma (1.6). The proof that these sequence all generate $I_{1}\left(T_{1}\right)_{P}=P\left(T_{1}\right) P$ for each minimal prime $P$ of $I_{1}$ reduces to studying the vector space $V=P\left(T_{1}\right)_{P} / P^{2}\left(T_{1}\right)_{P}$. One needs to know that given two sets of generators for this vector space, one can take general linear combinations of the first set (i.e., with indeterminate coefficients), say $v_{1}, \ldots, v_{k}$ of them, together with general linear combinations of the second, and if the number of these is $\operatorname{dim} V$, they will be a basis. The point is that the elements $v_{1}, \ldots, v_{k}$ form part of a basis. To decide whether the remaining elements complete the basis one may pass to $V / \operatorname{Span}\left\{v_{1}, \ldots, v_{k}\right\}$. Since we are working with elements that span this space, this is clear.

\section{THE MAP $\Phi$ AND THE MODULES $W_{S / R}$}

Our next main goal is to construct the maps $\Phi$ mentioned briefly in (2.5). Throughout this section we assume that $R$ is a normal Noetherian domain, with fraction field $\mathcal{K}$, that $S$ is a torsion-free generically étale $R$-algebra with total quotient ring $\mathcal{L}$ (by hypothesis, a finite product of separable field extensions of $\mathcal{K}$ ), that $T$ is a localization of the polynomial ring $R\left[X_{1}, \ldots, X_{n}\right]$ that maps onto $S$, with kernel $I$, and that the minimal primes of $I$ in $T$ are $P_{1}, \ldots, P_{r}$. We also assume that $S$ is relatively $\mathrm{S}_{1}$ presentable over $R$. However, in parts (c), (d) and (f) of Theorem (4.4), we shall need the stronger hypothesis that $S$ is relatively $\mathrm{S}_{2}$ presentable over $R$. Furthermore, we assume that $R \rightarrow S$ has arisen from $R_{0} \rightarrow S_{0}$ satisfying the same conditions by tensoring with $R=R_{0}(\mathcal{T})$ for some infinite family $\mathcal{T}$ of indeterminates, so that the results of Lemma (3.1) are applicable.

Lemma 5.1. Let $g_{1}, \ldots, g_{n} \in I$. Let $X$ denote $X_{1}, \ldots, X_{n}$. Then the images of the $g_{j}$ in $R[X]_{P_{\nu}}$ generate $P_{\nu} R[X]_{P_{\nu}}$ if and only if $\operatorname{det}\left(\partial g_{j} / \partial X_{i}\right) \notin P_{\nu}$. (The same holds if we only assume that $g_{1}, \ldots, g_{n} \in P_{\nu}$.) Hence, if $g_{1}, \ldots, g_{n}$ is a special sequence in $I$, then the image $\gamma$ of $\operatorname{det}\left(\partial g_{j} / \partial X_{i}\right)$ is not a zerodivisor in $S$, and so represents an invertible element of the total quotient ring $\mathcal{L}$ of $S$. 
Proof. The last statement clearly follows from the first, and we prove the first under the assumption that $g_{1}, \ldots, g_{n} \in P=P_{\nu}$. Let $\mathcal{M}$ be the maximal ideal corresponding to $P$ in $\mathcal{K}[X]$. Then $P R[X]_{P} \cong \mathcal{K}[X]_{\mathcal{M}}$, and so this is really a statement about when elements of $\mathcal{M}$ generate $\mathcal{M}$ in $\mathcal{K}[X]$. For the rest of this proof we shall write $\mathcal{L}$ (instead of, say, $\mathcal{L}_{\nu}$ ) for the residue field $R[X]_{P}$, which is the same as the residue field of $\mathcal{K}[X]_{\mathcal{M}}$. It is critical in what follows that $\mathcal{L}$ is separable over $\mathcal{K}$. Consider the universal $\mathcal{K}$-derivation $d: \mathcal{K}[X] \rightarrow \Omega_{\mathcal{K}[X] / \mathcal{K}}$, the module of Kähler differentials, which is the free module $\mathcal{K}[X]$-module generated by the elements $d X_{1}, \ldots, d X_{n}$. Of course, if $f \in \mathcal{K}[X]$ then $d f=\sum_{j=1}^{n}\left(\partial f / \partial x_{j}\right) d x_{j}$. The restriction of $d$ to $\mathcal{M}$ gives a $\mathcal{K}$-linear map $\mathcal{M} \rightarrow \Omega_{\mathcal{K}[X] / \mathcal{K}}$, and by the defining property of a derivation it sends $\mathcal{M}^{2} \rightarrow \mathcal{M} \Omega_{\mathcal{K}[X] / \mathcal{K}}$. Thus, there is an induced map of $\mathcal{K}$-vector spaces

$$
\delta: \mathcal{M} / \mathcal{M}^{2} \rightarrow \mathcal{L} \otimes_{\mathcal{K}[X]} \Omega_{\mathcal{K}[X] / \mathcal{K}}
$$

Both modules are $\mathcal{L}$-vector spaces and it follows from the defining property of a derivation that $\delta$ is actually $\mathcal{L}$-linear. Since $\mathcal{K}[X]_{\mathcal{M}}$ is regular of dimension $n$, $\mathcal{M} / \mathcal{M}^{2}$ is an $n$-dimensional vector space over $\mathcal{L}$. The key point is that under the hypothesis that $\mathcal{L}$ is separable over $\mathcal{K}$, the map $\delta$ is an isomorphism of $\mathcal{L}$-vector spaces. This is well known, but we give a very short proof. The map $\delta$ sends the elements represented by generators $g_{1}, \ldots, g_{n}$ for $\mathcal{M}$ to the elements represented by the $d g_{j}$, and so it has a matrix which is the image of the matrix $\left(\partial g_{j} / \partial x_{i}\right)$ after mapping the entries to $\mathcal{L}$. Thus, $\delta$ is an isomorphism if and only if the Jacobian determinant $\operatorname{det}\left(\partial g_{j} / \partial x_{i}\right)$ has nonzero image in $\mathcal{L}$. But this determinant generates $J_{\mathcal{L} / \mathcal{K}}$, and so $\delta$ is an isomorphism if and only if the Jacobian ideal of $\mathcal{L}$ over $\mathcal{K}$ is $\mathcal{L}$. But we may use any presentation of $\mathcal{L}$ over $\mathcal{K}$ to calculate $J_{\mathcal{L} / \mathcal{K}}$, and so we may instead use $\mathcal{L} \cong \mathcal{K}[Z] / f(Z)$ where $Z$ here represents just one variable and where $f$ is a single separable polynomial. The Jacobian determinant is then the value of $f^{\prime}(Z)$ in $L$, which is not zero by virtue of the separability.

Thus, $\delta$ is an $\mathcal{L}$-isomorphism. Moreover, we have already seen that if $g_{1}, \ldots, g_{n}$ are generators of $\mathcal{M}$ then the Jacobian determinant is not 0 in $\mathcal{L}$. But the converse is also clear, because if $g_{1}, \ldots, g_{n}$ are any elements of $\mathcal{M}$, they generate $\mathcal{M}$ if and only if their images in $\mathcal{M} / \mathcal{M}^{2}$ span this vector space over $\mathcal{L}$, by Nakayama's lemma, and this will be the case if and only if their further images in $\mathcal{L} \otimes_{\mathcal{K}[X]} \Omega_{\mathcal{K}[X] / \mathcal{K}}$ span that vector space over $\mathcal{L}$, since $\delta$ is an isomorphism, i.e., if and only if the images of the $d g_{j}$ span. But this is equivalent to the assertion that the images of the columns of the matrix $\left(\partial g_{j} / \partial x_{i}\right)$, after the entries are mapped to $\mathcal{L}$, span an $n$-dimensional space, i.e., to the nonvanishing of $\operatorname{det}\left(\partial g_{j} / \partial x_{i}\right)$ in $\mathcal{L}$, which is, of course, equivalent to its not being in $P$.

(4.2) The definition of $\theta$ and $\boldsymbol{W}_{S / \boldsymbol{R}}$ We continue the conventions in the first paragraph of this section, but because we shall let both $S$ and its presentation vary we shall write $\theta$ for the map $T \rightarrow S$ and we shall denote by $g$ a special sequence $g_{1}, \ldots, g_{n}$ in $I$. We may then temporarily define

$$
\Phi_{\theta, g}: \frac{\left.\left(g_{1}, \ldots, g_{n}\right) T:{ }_{T} I\right)}{\left(g_{1}, \ldots, g_{n}\right) T} \rightarrow \mathcal{L}
$$


by sending the class of $u$ to $\bar{u} / \gamma$ where $\bar{u}$ is the image of $u$ in $\mathcal{L}$, and $\gamma$ is the image of $\operatorname{det}\left(\partial g_{j} / \partial x_{i}\right)$ in $\mathcal{L}$ : the element $\gamma$ is invertible in $\mathcal{L}$ by Lemma (4.1). We shall often write $\Phi$ when $\theta$ and $g$ are understood. We shall soon show that the image of $\Phi$ is contained in $S:{ }_{\mathcal{L}} J_{S / R}$. Once this is established we shall change the definition of $\Phi$ very slightly by restricting its range to be $S:{ }_{\mathcal{L}} J_{S / R} \subseteq \mathcal{L}$.

We note that $\left(\left(g_{1}, \ldots, g_{n}\right) T:{ }_{T} I\right) /\left(g_{1}, \ldots, g_{n}\right) T \cong \operatorname{Hom}_{T}\left(T / I, T /\left(g_{1}, \ldots, g_{n}\right) T\right)$.

We shall denote the image of $\Phi_{\theta, g}$ in $\mathcal{L}$ by $W_{S / R}(\theta, g)$. However, we shall see just below that it is independent of the choices of $\theta$ and $g$, and once we know this we shall simply write it as $W_{S / R} \subseteq \mathcal{L}$.

Lemma 5.2. The map $\Phi_{\theta, g}$ is injective, and its image in $\mathcal{L}$ is independent of the choice of $g$, and of the choice of $\theta$. Its image is contained in $S:{ }_{\mathcal{L}} J_{S / R}$.

Proof. The domain of $\Phi$ may be thought of as the submodule of $T /\left(g_{1}, \ldots, g_{n}\right) T$ consisting of elements killed by $I$. Since $g_{1}, \ldots, g_{n}$ is a special sequence, the associated primes of $g_{1}, \ldots, g_{n}$ are all minimal, and it suffices to show that the map is injective after localizing at each minimal prime of $\left(g_{1}, \ldots, g_{n}\right) T$. If the prime does not contain $I$ the domain of $\Phi$ becomes 0 after localization and there is nothing to prove. If the prime contains $I$ it is one of the $P_{\nu}$, and after localization the map becomes the composition of the isomorphism $T_{P_{\nu}} / P_{\nu} T_{P_{\nu}} \cong \mathcal{L}_{P_{\nu}}$ with the automorphism of $\mathcal{L}_{P_{\nu}}$ induced by multiplication by the image of $\gamma$, which is nonzero.

To prove for a fixed presentation that the map is independent of the choice of special sequence suppose that we have two special sequences that yield maps with different images. We can preserve the fact that the images are different while localizing at a suitable prime or even maximal ideal of $T: S$ is replaced by its localization at a corresponding prime. Thus, there is no loss of generality in assuming that $T$ and $S$ are local. The sequences in question remain special as we localize. But we may now apply Lemma (3.1) to conclude the existence of a finite chain of special sequences joining the two that we are comparing such that any two consecutive sequences differ either in at most one spot, or by the action of an invertible matrix over $R$. Thus, we need only make the comparison when the two sequences differ in just one term, and since the sequences are permutable we may assume without loss of generality that one of them is $g_{1}, \ldots, g_{n}$ and the other is $h_{1}, \ldots, h_{n}$ where $h_{j}=g_{j}$ for $j \geqslant 2$. We set up an isomorphism

$$
\sigma: \frac{\left(g_{1}, \ldots, g_{n}\right) T:{ }_{T} I}{\left(g_{1}, \ldots, g_{n}\right) T} \cong \frac{\left(h_{1}, \ldots, h_{n}\right) T:{ }_{T} I}{\left(h_{1}, \ldots, h_{n}\right) T}
$$

as follows. If $u$ is an element of $\left(g_{1}, \ldots, g_{n}\right) T:{ }_{T} I$ then since $u I \subseteq\left(g_{1}, \ldots, g_{n}\right) T$ we may write $u h_{1}=v g_{1}+w$, where $w \in\left(g_{2}, \ldots, g_{n}\right) T=\left(h_{2}, \ldots, h_{n}\right) T$. We map the class of $u$ to the class of $v$. Note that any such $v$ is automatically in $\left(h_{1}, \ldots, h_{n}\right) T:{ }_{T} I$. (If $a \in I$ then $a v g_{1}+a w=a u h_{1}$. Now, $a u=b g_{1}+w^{\prime}$ where $w^{\prime} \in\left(g_{2}, \ldots, g_{n}\right) T$ and so $a v g_{1}+a w=b g_{1} h_{1}+w^{\prime} h_{1}$ and so $g_{1}\left(a v-b h_{1}\right)=$ $w^{\prime} h_{1}-a w \in\left(g_{2}, \ldots, g_{n}\right) T$. Since $g_{1}$ is not a zerodivisor on $\left(g_{2}, \ldots, g_{n}\right) T$, we have that $a v-b h_{1} \in\left(g_{2}, \ldots, g_{n}\right) T$, and so $a v \in\left(h_{1}, g_{2}, \ldots, g_{n}\right) T=\left(h_{1}, \ldots, h_{n}\right) T$, as required, for all $a \in I$.) Next note that the choice of $v$ given $u$ is unique modulo $\left(g_{2}, \ldots, g_{n}\right) T$. Thus, we have defined a map from $\left(g_{1}, \ldots, g_{n}\right) T:{ }_{T} I$ to 
$\left(\left(h_{1}, \ldots, h_{n}\right) T:{ }_{T} I\right) /\left(h_{1}, \ldots, h_{n}\right) T$. It is easy to see that this map kills $\left(g_{1}, \ldots, g_{n}\right) T$ and so we have a map as required. It is also clear that if we similarly define a map

$$
\frac{\left(h_{1}, \ldots, h_{n}\right) T:_{T} I}{\left(h_{1}, \ldots, h_{n}\right) T} \rightarrow \frac{\left(g_{1}, \ldots, g_{n}\right) T:_{T} I}{\left(g_{1}, \ldots, g_{n}\right) T}
$$

it will be an inverse for the map already constructed.

To complete the proof of the independence of the image from the choice of special sequence we note that the following diagram commutes:

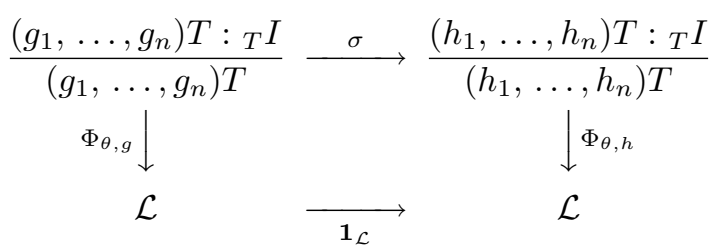

To see this, one simply needs to see that if

$$
u h_{1}-v g_{1}=\sum_{j=2}^{n} t_{j} g_{j}
$$

in $T$, then $\bar{u} / \gamma=\bar{v} / \eta$ in $c L$, where $\gamma, \eta$ are the respective images of $\operatorname{det}\left(\partial g_{j} / \partial X_{i}\right)$ and $\operatorname{det}\left(\partial h_{j} / \partial X_{i}\right)$ in $\mathcal{L}$, i.e., that $u \operatorname{det}\left(\partial h_{j} / \partial X_{i}\right) \equiv v \operatorname{det}\left(\partial g_{j} / \partial X_{i}\right)$ modulo $I$. By differentiating $(*)$ with respect to each $X_{j}$ in turn and using the fact that all the $g_{j}$ and $h_{j}$ are in $I$, we see that

$$
u \nabla h_{1}-v \nabla g_{1} \equiv \sum_{j=2}^{n} t_{j} \nabla g_{j} \quad \text { modulo } I
$$

which implies $(*)$.

For a given special sequence $g$ it is obvious from the definition of $\Phi_{\theta, g}$ that $\gamma$ multiplies the image of $\Phi_{\theta, g}$ into $S \subseteq \mathcal{L}$. Since the image is independent of the choice of special sequence, and since by Lemma (3.1) as the special sequence varies the values of $\gamma$ generate $J_{S / R}$, it follows that the image is contained in $S:{ }_{\mathcal{L}} J_{S / R}$.

It remains only to prove that the image of $\Phi_{T, g}$ is independent of the choice of $\theta: T \rightarrow S$ as well. We first consider the case of a finitely generated $R$-algebra $S$. The choice of a presentation is equivalent to the choice of a finite set of generators for $S$ over $R$. We can compare the results from each of two different presentations with the result from their union, and so it suffices to see what happens when we enlarge a set of generators. By induction, it suffices to show that the image does not change when we enlarge a set of generators by one element, and so we may assume that we have $\theta: T=R\left[X_{1}, \ldots, X_{n}\right] \rightarrow S$ and an extension of $\theta, \theta^{\prime}: T\left[X_{n+1}\right] \rightarrow S$ by sending $X_{n+1}$ to $s$. Let $T^{\prime}=T\left[X_{n+1}\right]$. We can choose an element $F \in T$ such that $F$ maps to $s$ in $S$, and it follows easily that the kernel $I^{\prime}$ of $\theta^{\prime}$ is $I+\left(X_{n+1}-F\right)$. It also follows easily that if $g=g_{1}, \ldots, g_{n}$ is special in $I$ then $g^{\prime}=g_{1}, \ldots, g_{n+1}$ with $g_{n+1}=X_{n+1}-F$ is a special sequence in $I^{\prime}$. The larger (size $\left.n+1\right)$ Jacobian matrix has the same determinant $\gamma$ as the size $n$ Jacobian matrix of $g_{1}, \ldots, g_{n}$ with 
respect to $X_{1}, \ldots, X_{n}$, and it is easy to check that there is an isomorphism

$$
\tau: \frac{\left(g_{1}, \ldots, g_{n}\right) T:{ }_{T} I}{\left(g_{1}, \ldots, g_{n}\right) T} \cong \frac{\left(g_{1}, \ldots, g_{n+1}\right) T^{\prime}: T^{\prime} I^{\prime}}{\left(g_{1}, \ldots, g_{n+1}\right) T^{\prime}}
$$

which is induced by the inclusion $\left(g_{1}, \ldots, g_{n}\right) T:{ }_{T} I \subseteq\left(g_{1}, \ldots, g_{n+1}\right) T^{\prime}:{ }_{T} I^{\prime}$. Since the Jacobian determinants are the same we have a commutative diagram

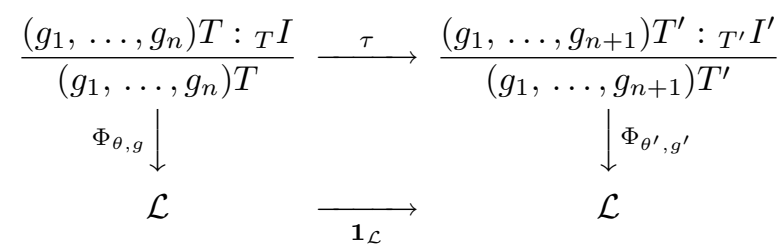

and this yields that the images are the same.

We have now justified the notation $W_{S / R}$ when $S$ is finitely generated over $R$. We leave it to the reader to verify that if $s$ is a nonzerodivisor in $S$, then $W_{S\left[s^{-1}\right] / R}=$ $\left(W_{S / R}\right)_{s}$, and that $W_{S / R}(\theta)$ is independent of $\theta$ when $S$ is essentially of finite type over $R$.

Proposition 5.3. Proposition (4.4) Let $S$ be generically étale, torsion-free and essentially of finite type over the Noetherian normal domain $R$. Assume that $S$ is relatively $S_{1}$ presentable over $R$. Let $W=W_{S / R}$.

(a) For any multiplicative system $U$ in $S, W_{U^{-1} S / R}=U^{-1} W$.

(b) $W$ is torsion-free over $S$.

For the remaining parts, assume that $S$ is relatively $S_{2}$ presentable over $R$ (automatic in part (e), since $R$ is regular).

(c) For every prime ideal $P$ of $S$, if $u, v$ is part of a system of parameters for $S_{P}$ then it is a regular sequence on $W_{P}$. (Thus, $W$ is $S_{2}$.)

(d) If $W \subseteq W^{\prime} \subseteq \mathcal{L}$ and $W_{P}=W_{P}^{\prime}$ for all height one primes of $S$ and for all minimal primes of $S$ that are also maximal ideals, then $W=W^{\prime}$.

(e) If $R \rightarrow S$ is a local homomorphism of regular local rings then $J_{S / R}$ is principal and $W=S:{ }_{\mathcal{L}} J_{S / R}$.

(f) If $S$ is normal and $R_{P}$ is regular for every prime ideal $P$ of $R$ lying under a height one prime ideal $Q$ of $S$, then $W=S:{ }_{\mathcal{L}} J_{S / R}$.

Proof. Part (a) is essentially the last part of (4.3), while (b) is evident from the fact that $W \subseteq \mathcal{L}$, by definition.

To prove (c) note that by (a) we may assume that $S$ is local and that $u, v$ is part of a system of parameters. We may choose a presentation $\theta: T \rightarrow S$ and think of $W$ as $\cong\left(\left(g_{1}, \ldots, g_{n}\right) T:{ }_{T} I\right) /\left(g_{1}, \ldots, g_{n}\right) T$, where the sequence $g_{1}, \ldots, g_{n}$ is not only special, but has the additional property that $T /\left(g_{1}, \ldots, g_{n}\right)$ is $\mathrm{S}_{2}$. Let $u_{0}, v_{0} \in T$ be representatives of $u, v$. Then $u_{0}+I$ cannot be contained in the union of the associated primes of $\left(g_{1}, \ldots, g_{n}\right)$ (these are the same as the minimal primes), or else it will be contained in one of them by [Kap], Theorem 124. Since this will contain $I$, it will be a minimal prime of $I$, and contradicts the statement that $u$ is part of a system of parameters in $S=T / I$. Thus, we can replace $u_{0}$ by an element $u_{1}$ representing $u$ such that $g_{1}, \ldots, g_{n}, u_{1}$ is a regular sequence in $T$. Similarly, $v_{0}+I$ 
cannot be contained in the union of the associated primes of $\left(g_{1}, \ldots, g_{n}, u_{1}\right) T$, or else it is contained in one of them, say $Q$. Thinking modulo $I$, we see that, since $Q$ contains $u_{0}, v_{0}$, it has height at least two more than $I$. But then $Q$ has depth at least $n+2$, a contradiction, since it is supposedly an associated prime of an ideal generated by a regular sequence of length $n+1$. Thus, we may choose $u_{1}, v_{1}$ in $T$ representing $u, v$ respectively and such that $g_{1}, \ldots, g_{n}, u_{1}, v_{1}$ is a regular sequence. Clearly, $u_{1}, v_{1}$ form a regular sequence on $T /\left(g_{1}, \ldots, g_{n}\right) T$. We claim they also form a regular sequence on the set of elements killed by $I$. It is clear that $u_{1}$ remains not a zerodivisor on this set. Suppose that $v_{1} z=u_{1} y$ where $z, y$ are killed by $I$. Then $z=u_{1} x, y=-v_{1} x$ where, a priori, $x \in T /\left(g_{1}, \ldots, g_{n}\right) T$. But $I z=0$ and so $I u_{1} x=0$, and since $u_{1}$ is not a zerodivisor on $T /\left(g_{1}, \ldots, g_{n}\right) T$, it follows that $I x=0$ as well.

Part (d) is a standard consequence of what we proved in part (c), but we give a short argument. If $W \neq W^{\prime}$ we can localize at a minimal prime of the support of $W^{\prime} / W$ and preserve the counterexample. By hypothesis, this prime cannot have height one (nor height 0 , since if a height 0 prime is not maximal then we can localize at it in two steps: first localize at a height one prime that contains it). Thus, we may assume that $S$ is local of height two or more, and that $W^{\prime} / W$ is a nonzero module of finite length. It follows that we can choose an element $x \in W^{\prime}-W$ and part of a system of parameters $u, v$ for $S$ such that $u z$ and $v z$ are in $W$. The relations $v(u z)=u(v z)$ over $W$ together with part (c) show that $u z \in u W$, and it follows that $z \in W$ after all, a contradiction.

To prove (e) note that when $R$ is regular so is $T$, and so $T \rightarrow S$ will be a surjection of local rings. The kernel of such a surjection must be generated by part of a minimal set of generators for the maximal ideal of $T$. It follows that $I$ is a prime and we have $I=\left(g_{1}, \ldots, g_{n}\right) T$ is itself generated by a suitable special sequence. Then $J_{S / R}$ is generated by $\gamma=\operatorname{det}\left(\partial g_{j} / \partial X_{i}\right)$, and $\left(\left(g_{1}, \ldots, g_{n}\right) T:{ }_{T} I\right) /\left(g_{1}, \ldots, g_{n}\right) T=$ $\left(I:{ }_{T} I\right) / I=T / I=S$ and $\Phi$ sends 1 to $\frac{1}{\gamma}$, so that $W=S \frac{1}{\gamma}$, and one sees that $S:{ }_{\mathcal{L}} J_{S / R}=S:{ }_{\mathcal{L}} \gamma S=W$, as claimed.

To prove (f) it suffices by (d) to consider the problem after localizing at a height one or zero prime $Q$ of $S$, and, without affecting the issue, one may also localize $R$ at its contraction. If the prime of $S$ has height 0 , so does its contraction to $R$, and both rings become regular after localization. If the prime of $S$ has height one, then, again, both rings become regular after localization, $S$ because it is normal and $R$ by hypothesis. In either case the result follows from part (e).

\section{A CRITICAL LEMMA AND THE FINAL STEP OF THE PROOF}

The following result of Lipman and Sathaye is critical in establishing that $W_{S / R}$ decreases as $S$ is increased by adjoining integral fractions.

Lemma 6.1 (Lipman-Sathaye). Let $T$ be a commutative ring, $Y$ an indeterminate, and $J$ an ideal of $T[Y]$ such that $J$ contains a monic polynomial $h$ in $Y$ of degree $d$, and such that $J$ also contains an element of the form $\alpha Y-\beta$ where $\alpha, \beta \in T$ are such that $J:{ }_{T[Y]} \alpha T[Y]=J$, i.e., such that $\alpha$ is not a zerodivisor mod- 
ulo J. Let $G \subseteq T$ be an ideal of $T$ with $G \subseteq J$. Then for every element $v \in T[Y]$ such that $v J \subseteq(h, G) T[Y]$ there is a $u \in T$ such that $u(J \cap T) \subseteq G$ and such that $v \equiv u \frac{\partial h}{\partial Y}$ modulo $J$.

Proof. See Lemma (3.17) on p. 216 of [LS].

We now use this to prove:

Theorem 6.2. If $S_{1}$ is obtained from $S$ by adjoining finitely many integral fractions of $\mathcal{L}$, then $W_{S_{1} / R} \subseteq W_{S / R}$.

Proof. By induction on the number of fractions adjoined, it is obviously sufficient to prove this when $S_{1}=S[\lambda]$, where $\lambda$ is a single element of $\mathcal{L}$. Choose a presentation $\theta: T \rightarrow S$ and a special sequence $g_{1}, \ldots, g_{n}$ in the kernel $I$. Let $Y$ be a new indeterminate and extend $\theta$ to a map $T[Y] \rightarrow S[\lambda]$ by sending $Y$ to $\lambda$. Since $\lambda$ is integral over $S$ there is a monic polynomial $h=h(Y) \in T[Y]$ of degree say, $d$, in the kernel $J$ of $T[Y] \rightarrow S[\lambda]$. If $\lambda \in S$ there is nothing to prove so that we may assume that $d \geqslant 2$. Since $\lambda$ is in $\mathcal{L}$ we may also choose $\alpha$ and $\beta$ in $T$ with $\alpha$ not a zerodivisor on $I$ such that $\alpha Y-\beta$ is in the kernel. Consider the image of $h(Y)$ in $S[Y]$. There will be a certain subset of the minimal primes of $S$ such that the image of $\lambda$ is a multiple root of the image of $h$ modulo those primes. If that set of primes is empty, we shall not alter $h$. If it is not empty choose an element of $S$ that is not in any of those minimal primes but that is in the others, and represent it by an element $t \in T$. Then $h(Y)+t(\alpha Y-\beta)$ has the property that its image modulo any minimal prime of $S$ has the image of $\lambda$ as a simple root, and so we may assume, using this polynomial in place of the original choice of $h$, that $h$ is a monic polynomial of degree $d \geqslant 2$ such that image of $\lambda$ modulo every minimal prime of $\mathcal{L}$ is a simple root of the image of $h$.

Because $h$ is monic in $Y$, the sequence $g_{1}, \ldots, g_{n}, h$ is a regular sequence, and the Jacobian determinant with respect to $X_{1}, \ldots, X_{n}, Y$ is $\gamma \frac{\partial h}{\partial Y}$, where $\gamma$ is $\operatorname{det}\left(\partial g_{j} / \partial X_{i}\right)$. Our choice of $h$ implies that $\frac{\partial h}{\partial Y}$ has image that is not in any minimal prime of $\mathcal{L}$, and it follows, using Lemma (1.9), that $g_{1}, \ldots, g_{n}, h$ is a special sequence in $J$ and can be used to calculate $W_{S[\lambda] / R}$. Let $v \in\left(g_{1}, \ldots, g_{n}, h\right) T[Y]:{ }_{T[Y]} J$. We may now apply Lemma (5.1) with this $T, Y, J, v, \alpha, \beta$ and $h$, while taking $G=\left(g_{1}, \ldots, g_{n}\right) T$. Note that $J \cap T=I$. Now, $v$ gives rise to a typical element, the image of $v /\left(\gamma \frac{\partial h}{\partial Y}\right)$ in $\mathcal{L}$, in $W_{S[\lambda] / R}$, and we want to show that this element is in $W_{S / R}$. Pick $u$ as in Lemma (5.1). Then $u \in\left(g_{1}, \ldots, g_{n}\right) T:{ }_{T} I$ and since $v \equiv u \frac{\partial h}{\partial y}$ modulo $J$, this image is the same as the image of $\left(u \frac{\partial h}{\partial y}\right) /\left(\gamma \frac{\partial h}{\partial y}\right)=u / \gamma$, and so is in $W_{S / R}$, as required.

The proof of the main theorem. Theorem (5.2) and Proposition (4.4f) justify the argument given much earlier in (2.6), and the proof of Theorem (2.1) is now complete. 


\section{References}

[1] J. Briançon and H. Skoda, Sur la clôture intégrale d'un idéal de germes de fonctions holomorphes en un point de $C^{n}$, C.R. Acad. Sci. Paris Sér. A, 278(1974), 949-951.

[2] W. Bruns Tight closure, Bull. Amer. Math. Soc., 33(1996), 447-458.

[3] M. Hochster, Properties of Noetherian rings stable under general grade reduction, Arch. Math. 24(1973), 393-396.

[4] __- Tight closure in equal characteristic, big Cohen-Macaulay algebras, and solid closure in Commutative Algebra: Syzygies, Multiplicities and Birational Algebra, Contemp. Math. 159, Amer. Math. Soc., Providence, R. I., 1994, 173-196.

[5] _-_-_, The notion of tight closure in equal characteristic zero in Proc. of the CBMS Conference on Tight Closure and Its Applications (Fargo, North Dakota, July, 1995), Appendix to the notes on the main lectures by Craig Huneke, C.B.M.S. Regional Conference Series, Amer. Math. Soc., Providence, R.I., 1996.

[6] M. Hochster and C. Huneke, Tightly closed ideals, Bull. Amer. Math. Soc.18(1988), 45-48.

[7] _-_ Tight closure in Commutative Algebra, Math. Sci. Research Inst. Publ., Springer-Verlag, New York-Berlin-Heidelberg, 15(1989), 305-324.

[8] --_---- , Tight closure and strong F-regularity, Mémoires de la Société Mathématique de France, numéro 38 (1989), 119-133.

[9] _-__._, Tight closure, invariant theory, and the Briançon-Skoda theorem, J. Amer. Math. Soc. 3(1990), 31-116.

[10] -_-_-_, Infinite integral extensions and big Cohen-Macaulay algebras, Annals of Math. 135(1992), 53-89.

[11] -_- F-regularity, test elements, and smooth base change, Trans. Amer. Math. Soc. 346(1994), 1-62.

[12] -------, Localization and test exponents for tight closure, Michigan Math. J. 48(2000), 305-329.

[13] --_----, Tight closure in equal characteristic zero, preliminary preprint.

[14] C. Huneke, Tight Closure and Its Applications, Proc. of the CBMS Conference held at Fargo, North Dakota, July, 1995, C.B.M.S. Regional Conference Series, Amer. Math. Soc., Providence, R.I., 1996.

[15] I. Kaplansky, Commutative rings, Revised Edition, The Univ. of Chicago Press, Chicago and London, 1974.

[16] J. Lipman and A. Sathaye, Jacobian ideals and a theorem of BriançonSkoda, Michigan Math. J. 28(1981), 199-222.

[17] J. Lipman and B. Teissier, Pseudo-rational local rings and a theorem of Briançon-Skoda about integral closures of ideals, Michigan Math. J. 28(1981), 97-116. 
[18] H. Matsumura, Commutative Algebra, W.A. Benjamin, New York, 1970.

[19] D. Rees, A note on analytically unramified local rings, J. London Math. Soc. 36(1961), 24-28.

[20] H. Skoda, Applications des techniques $L^{2}$ a la théorie des idéaux d'une algébre de fonctions holomorphes avec poids, Ann. Scient. Ec. Norm. Sup. 4éme série, t. 5(1972), 545-579.

This article may be accessed via WWW at http://www.rmi.acnet.ge/hha/ or by anonymous ftp at

ftp://ftp.rmi.acnet.ge/pub/hha/volumes/2002/n2a13/v4n2a13.(dvi,ps,pdf)

Melvin Hochster hochster@math.1sa.umich.edu

Department of Mathematics

University of Michigan

Ann Arbor, MI 48109-1109

USA 